\title{
Perfil e sobrecarga dos cuidadores informais de idosos dependentes
}

\author{
Profile and overload of informal caregivers of dependent elderly people \\ Perfil y sobrecarga de cuidadores informales de personas mayores dependientes
}

Recebido: 13/05/2021 | Revisado: 19/05/2021 | Aceito: 21/05/2021 | Publicado: 07/06/2021

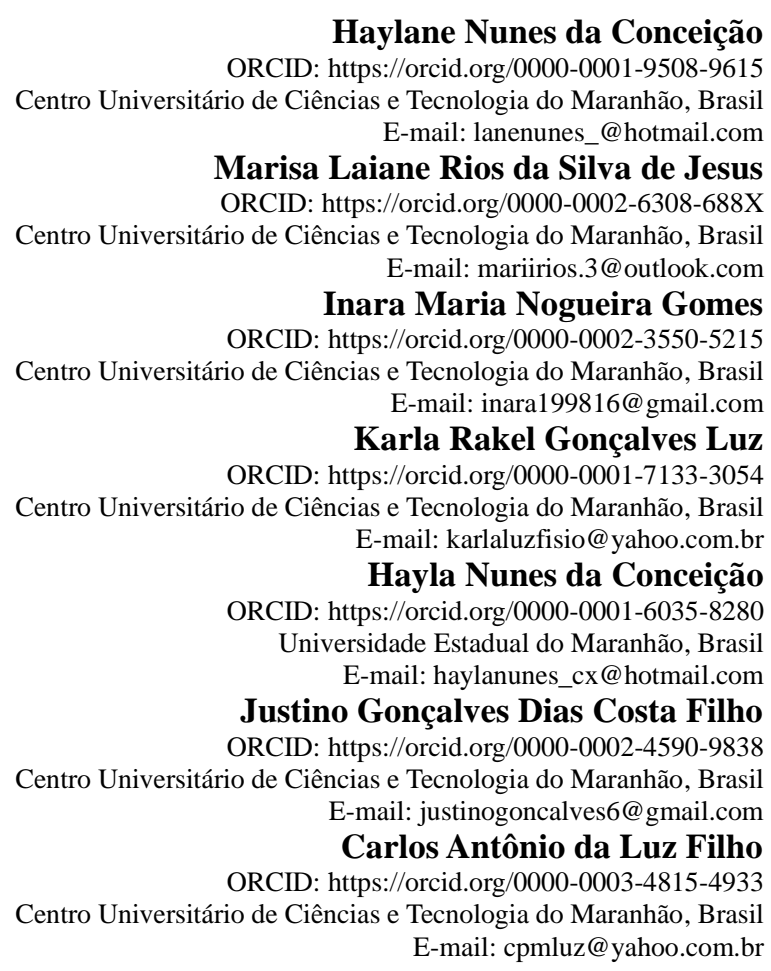

\section{Resumo}

Objetivo: Avaliar o perfil e a sobrecarga dos cuidadores informais de idosos dependentes. Metodologia: Trata-se de um estudo transversal e descritivo, realizado com os cuidadores informais de idosos dependentes cadastrados na área de abrangência de quatro Unidades Básicas de Saúde do município de Caxias, Maranhão. Os dados foram coletados no período de junho a outubro de 2019, por meio de um questionário elaborado pelos pesquisadores para a caracterização dos participantes e pela aplicação da versão brasileira da escala de Zarit Burder Interview. Resultados: A população do estudo foi composta por 52 cuidadores. A função de cuidador foi exercida mais frequentemente por cuidadores do sexo feminino $(n=47 ; 90,4 \%)$, na faixa etária entre 41 a 50 anos de idade $(n=21 ; 40,4 \%)$, casados $(n=32 ; 61,3 \%)$, com ensino fundamental incompleto $(n=23 ; 44,2 \%)$, sendo os filhos $(n=22 ; 42,3 \%)$ os principais responsáveis pelo cuidado. A maioria dos cuidadores exerciam a função por mais de quatro anos $(\mathrm{n}=19 ; 36,5 \%)$, dedicando mais de oito horas diárias de assistência ao idoso $(\mathrm{n}=22 ; 42,3 \%)$ e não recebiam apoio de outras pessoas no cuidado $(\mathrm{n}=49 ; 94,2 \%)$. Constatou-se ainda que grande parte dos cuidadores apresentavam algum nível de sobrecarga (n=47; 90,4\%). Conclusão: As informações referentes ao perfil e o nível de sobrecarga dos cuidadores informais poderão sensibilizar os profissionais da Atenção Básica do município sobre os impactos do cuidado ao idoso dependente na saúde de seus cuidadores, contribuindo para o planejamento de estratégias que assegurem a qualidade de vida e a saúde desta população.

Palavras-chave: Cuidador; Idoso; Atenção primária à saúde.

\section{Abstract}

Objective: To assess the profile and burden of informal caregivers of dependent elderly people. Methodology: This is a cross-sectional and descriptive study, carried out with informal caregivers of dependent elderly people registered in the area covered by four Basic Health Units in the city of Caxias, Maranhão. Data were collected from June to October 2019, through a questionnaire prepared by the researchers to characterize the participants and by applying the Brazilian version of the Zarit Burder Interview scale. Results: The study population consisted of 52 caregivers. The role of caregiver was most often exercised by female caregivers $(n=47 ; 90.4 \%)$, aged 41 to 50 years old $(n=21$; $40.4 \%)$, married $(\mathrm{n}=32 ; 61.3 \%)$, with incomplete elementary education $(\mathrm{n}=23 ; 44.2 \%)$, with children $(\mathrm{n}=22$; 
42.3\%) being the main caregivers. Most caregivers performed the function for more than four years $(n=19 ; 36.5 \%)$, dedicating more than eight hours of daily assistance to the elderly $(\mathrm{n}=22 ; 42.3 \%)$ and did not receive support from other people in care $(n=49 ; 94.2 \%)$. It was also found that most caregivers had some level of burden $(n=47 ; 90.4 \%)$. Conclusion: Information regarding the profile and level of burden of informal caregivers may raise the awareness of Primary Care professionals in the municipality about the impacts of care for dependent elderly people on the health of their caregivers, contributing to the planning of strategies that ensure quality of life. and the health of this population.

Keywords: Caregivers; Aged; Primary health care.

\begin{abstract}
Resumen
Objetivo: Evaluar el perfil y la carga de los cuidadores informales de ancianos dependientes. Metodología: Se trata de un estudio transversal y descriptivo, realizado con cuidadores informales de ancianos dependientes registrados en el área de cobertura de cuatro Unidades Básicas de Salud de la ciudad de Caxias, Maranhão. Los datos fueron recolectados de junio a octubre de 2019, a través de un cuestionario elaborado por los investigadores para caracterizar a los participantes y aplicando la versión brasileña de la escala Zarit Burder Interview. Resultados: La población de estudio estuvo conformada por 52 cuidadores. El rol de cuidador lo ejercían con mayor frecuencia mujeres cuidadoras $(\mathrm{n}=47 ; 90,4 \%)$, de 41 a 50 años $(\mathrm{n}=21 ; 40,4 \%)$, casadas $(\mathrm{n}=32 ; 61,3 \%)$, con educación primaria incompleta $(\mathrm{n}=$ $23 ; 44,2 \%)$, siendo los niños $(n=22 ; 42,3 \%)$ los principales cuidadores. La mayoría de los cuidadores desempeñaron la función durante más de cuatro años $(n=19 ; 36,5 \%)$, dedicando más de ocho horas diarias de atención a los ancianos $(n=22 ; 42,3 \%)$ y no recibieron apoyo de otras personas a cargo $(n=49 ; 94,2 \%)$. También se encontró que la mayoría de los cuidadores tenían algún nivel de sobrecarga $(n=47 ; 90,4 \%)$. Conclusión: La información sobre el perfil y nivel de sobrecarga de los cuidadores informales puede sensibilizar a los profesionales de Atención Primaria del municipio sobre los impactos del cuidado de las personas mayores dependientes en la salud de sus cuidadores, contribuyendo a la planificación de estrategias que aseguren la calidad de vida y la salud de esta población.
\end{abstract}

Palabras clave: Cuidadores; Anciano; Atención primaria de salud.

\title{
1. Introdução
}

O Brasil, assim como outros países do mundo, vivencia o aumento acentuado da população idosa (Medeiros, Pinto Junior, Bousquat, \& Medina, 2017). A Pesquisa Nacional por Amostra de Domicílios (PNAD) revelou que o número de brasileiros com 60 anos ou mais representavam 9,8\% da população, em 2005, passando para 14,3\% em 2015 (Instituto Brasileiro de Geografia e Estatística [IBGE], 2016). Estimativas apontam que esse crescimento irá continuar nas próximas décadas, podendo ultrapassar, em 2060, o número de 58 milhões de idosos, o que corresponderá a 25,49\% da população brasileira (IBGE, 2010).

À medida que os anos de vida da população aumentam, ocorre, paralelamente, uma redução progressiva da capacidade funcional dos indivíduos, decorrente do declínio funcional e das perdas cognitivas da senescência e, frequentemente, dos problemas de saúde, especialmente, as doenças crônicas, que podem comprometem a autonomia do idoso, implicando a necessidade de um cuidador (Anjos et al., 2015; Pedreira \& Oliveira, 2012).

O cuidador de idosos, ocupação reconhecida pela Classificação Brasileira de Ocupações (CBO), é o indivíduo responsável por facilitar a execução das atividades de vida diária (AVDs) e assegurar a saúde, educação, cultura e bem-estar dos idosos por ele assistidos (Couto, Castro \& Caldas, 2016; Batista, Almeida \& Lancman, 2014).

Os cuidadores podem ser classificados como formais ou informais. Os cuidadores formais são indivíduos de 18 anos ou mais, com ensino fundamental completo, cursos livres de 80/160 horas e que recebem remuneração pelos serviços prestados, enquanto que os cuidadores informais, não possuem nenhum curso de capacitação e trabalham voluntariamente. Os cuidadores informais são membros da comunidade, amigos ou familiares, sendo a forma mais frequente de assistência ao idoso (Batista et al., 2014; Maronesi, Silva, Cantu \& Santos, 2014; Mendes, Figueiredo, Santos, Fernandes, Fonseca, 2019).

O suporte informal de cuidado pode ocasionar desgaste físico, ansiedade e sobrecarga nos cuidadores, uma vez que esses indivíduos não estão devidamente preparados para atender as complexas demandas que envolvem o cuidado a um idoso dependente (Diniz et al., 2018; Fernandes et al., 2013). Neste cenário, conhecer as características e o nível de sobrecarga dos cuidadores informais poderá favorecer o planejamento de estratégias que minimizem os impactos na saúde desta população. Desta maneira, o presente estudo buscou avaliar o perfil e a sobrecarga dos cuidadores informais de idosos dependentes. 


\section{Metodologia}

Trata-se de um estudo transversal e descritivo, realizado com os cuidadores informais de idosos dependentes cadastrados na área de abrangência de quatro Unidades Básicas de Saúde (UBS) do município de Caxias, Maranhão, sendo elas: UBS Cohab, UBS Baixinha, UBS Mutirão e UBS Volta Redonda. As UBS foram selecionadas pelos seguintes critérios de conveniência: adesão da coordenação da unidade em participar da pesquisa e disponibilidade dos Agentes Comunitários de Saúde (ACS) em acompanharem os pesquisadores na coleta.

Foram incluídos todos os cuidadores informais de idosos dependentes de ambos os sexos, com idade igual ou superior a 18 anos e que exerciam a função de cuidador principal por pelo menos seis meses. Foram excluídos os cuidadores formais e os cuidadores secundários dos idosos. Todos os participantes assinaram o termo de consentimento livre e esclarecido. Dos 59 cuidadores informais elegíveis para a pesquisa, 7 recusaram fazer parte do estudo, resultando em uma amostra de 52 cuidadores.

A coleta de dados foi realizada por um pesquisador treinado e feita através de uma entrevista, em sessão única e no domicílio dos idosos, no período de junho a outubro de 2019, durante as visitas domiciliares dos ACS de cada UBS. Todas as entrevistas foram feitas em um local reservado, sem a presença do idoso, a fim de evitar interferências nas respostas e para que os participantes se sentissem confortáveis para responder os questionamentos.

Os instrumentos utilizados para a coleta foram um questionário para a caracterização do cuidador e a versão brasileira da escala de Zarit Burder Interview (ZBI). O questionário foi elaborado previamente pelos pesquisadores, sendo composto por variáveis sociodemográficas e relacionadas ao cuidado. As variáveis sociodemográficas incluíram o sexo (masculino e feminino), estado civil (solteiro(a), casado(a), divorciado(a), viúvo (a), faixa etária ( $\geq 18$ e $\leq 20 ; 21$ a 30; 31 a 40; 41 a 50; 51 a 60; > 60), escolaridade (Não alfabetizado, Ensino fundamental incompleto, Ensino fundamental completo, Ensino médio incompleto, Ensino médio completo, Ensino superior incompleto, Ensino superior completo) e grau de parentesco com o idoso (cônjuge, filho(a), neto(a), irmão(a), outro parente, sem parentesco). Os cuidadores também foram questionados sobre o tempo que exercem a função de cuidador (menos de 1 ano, 1 a 2 anos, 3 a 4 anos, mais de 4 anos), tempo diário dedicado ao cuidado (menos de 4 horas, 4 a 6 horas, 6 a 8 horas, mais de 8 horas) e sobre o apoio de outras pessoas no cuidado (sim e não).

A escala ZBI é um instrumento composto por 22 questões utilizado para avaliar a sobrecarga subjetiva e objetiva dos cuidadores. Cada item avaliado pode pontuar de 0 a 4, perfazendo uma pontuação final que varia de 0 a 88 pontos. Este escore permite classificar a sobrecarga da seguinte forma: ausência de sobrecarga (0 a 20), sobrecarga leve a moderada (21 a 40), sobrecarga moderada a severa (41 a 60) e sobrecarga intensa (61 a 88) (Boaventura, Borges \& Ozaki, 2016; Costa et al., 2019).

Após a coleta, os dados foram tabulados no programa Microsoft Excel 2019, com digitação dupla. Em seguida, os dados foram exportados para o Statistical Package for the Social Sciences (SPSS) versão 20.0, onde foram analisados. As informações foram analisadas descritivamente por meio de frequência absolutas e relativas.

Este estudo originou-se de um projeto maior, intitulado "Cuidando do cuidador: relação da presença de sintomas osteomusculares com o trabalho dos cuidadores de idosos dependentes" que foi submetido ao Comitê de Ética e Pesquisa da Faculdade de Ciências e Tecnologia do Maranhão (FACEMA) e aprovado pelo parecer $\mathrm{n}^{\circ} 3367030$ e CAAE: 13252019.4.0000.8007.

\section{Resultados}

A caracterização dos cuidadores informais de idosos dependentes encontra-se descrita na Tabela 1. A função de cuidador foi mais frequentemente realizada por cuidadores do sexo feminino $(n=47 ; 90,4 \%)$, casados $(n=32 ; 61,3 \%)$ e com idade entre 41 a 50 anos $(n=21 ; 40,4 \%)$. Em relação a escolaridade, observou-se que a maior parte dos cuidadores apresentavam baixo nível de instrução, com 44,2\% (n=23) possuindo apenas o ensino fundamental incompleto. Quanto ao grau 
de parentesco, foi identificado que a maioria dos participantes apresentavam algum vínculo familiar com o idoso, sendo que 42,3\% ( $n=22)$ eram filhos, $28,8 \%(n=15)$ netos, 9,6\% (n=5) cônjuge, 7,7\% (n=4) irmãos e 5,8\% (n=3) outro parente.

No que se refere ao tempo da função de cuidador, foi identificado que 36,5\% (n=19) desempenham essa ocupação há mais de quatro anos, com 42,3\% ( $\mathrm{n}=22)$ dedicando mais de oito horas de cuidados diários ao idoso dependente. Observou-se ainda que a maioria dos cuidadores, $94,2 \%(\mathrm{n}=49)$ não recebem apoio de outras pessoas durante os cuidados ao idoso (Tabela $1)$.

Tabela 1. Perfil dos cuidadores informais de idosos dependentes em Caxias, Maranhão entre junho a outubro de 2019. Caxias, MA, Brasil, 2019.

\begin{tabular}{|c|c|c|}
\hline VARIÁVEIS & $\mathbf{N}$ & $\%$ \\
\hline \multicolumn{3}{|l|}{ Sexo } \\
\hline Feminino & 47 & 90,4 \\
\hline Masculino & 5 & 9,6 \\
\hline \multicolumn{3}{|l|}{ Faixa etária } \\
\hline$\geq 18$ e $\leq 20$ & 4 & 7,7 \\
\hline 21 a 30 & 5 & 9,6 \\
\hline 31 a 40 & 7 & 13,5 \\
\hline 41 a 50 & 21 & 40,4 \\
\hline 51 a 60 & 12 & 23,1 \\
\hline$>60$ & 3 & 5,8 \\
\hline \multicolumn{3}{|l|}{ Estado civil } \\
\hline Solteiro(a) & 9 & 17,3 \\
\hline Casado(a) & 32 & 61,3 \\
\hline Divorciado(a) & 8 & 15,4 \\
\hline Viúvo(a) & 3 & 5,8 \\
\hline \multicolumn{3}{|l|}{ Escolaridade } \\
\hline Não alfabetizado & 5 & 9,6 \\
\hline Ensino fundamental incompleto & 23 & 44,2 \\
\hline Ensino fundamental completo & 9 & 17,3 \\
\hline Ensino médio incompleto & 6 & 11,5 \\
\hline Ensino médio completo & 5 & 9,6 \\
\hline Ensino superior incompleto & 2 & 3,8 \\
\hline Ensino superior completo & 2 & 3,8 \\
\hline \multicolumn{3}{|l|}{ Grau de parentesco } \\
\hline Cônjuge & 5 & 9,6 \\
\hline Filho(a) & 22 & 42,3 \\
\hline Neto (a) & 15 & 28,8 \\
\hline Irmão(a) & 4 & 7,7 \\
\hline Outro parente & 3 & 5,8 \\
\hline Sem parentesco & 3 & 5,8 \\
\hline \multicolumn{3}{|c|}{ Tempo que exerce a função de cuidador } \\
\hline menos de 1 ano & 8 & 15,4 \\
\hline 1 a 2 anos & 13 & 25,0 \\
\hline 3 a 4 anos & 12 & 23,1 \\
\hline mais de 4 anos & 19 & 36,5 \\
\hline $\begin{array}{l}\text { Tempo diário dedicado } \\
\text { cuidado }\end{array}$ & & \\
\hline Menos de 4 horas & 4 & 7,7 \\
\hline De 4 a 5 horas & 10 & 19,2 \\
\hline De 6 a 7 & 16 & 30,8 \\
\hline Mais de 8 & 22 & 42,3 \\
\hline
\end{tabular}

Recebe apoio de outras pessoas no cuidado 


\begin{tabular}{lll}
\hline Sim & 3 & 5,8 \\
Não & 49 & 94,2 \\
\hline Total & $\mathbf{5 2}$ & $\mathbf{1 0 0}$ \\
\hline
\end{tabular}

Fonte: Autores (2019).

A avaliação da sobrecarga dos cuidadores, demonstrou que 90,4\% (n=47) dos participantes relataram algum nível de sobrecarga, sendo que 40,4\% ( $n=21)$ apresentaram uma sobrecarga intensa, $32,7 \%(n=17)$ uma moderada a severa e $17,3 \%$ $(n=9)$ uma leve a moderada. Cinco $(9,6 \%)$ cuidadores revelaram ausência de sobrecarga (Tabela 2).

Tabela 2. Grau de sobrecarga dos cuidadores informais de idosos dependentes entre junho a outubro de 2019 em Caxias, Maranhão, segundo a Escala ZBI. Caxias, MA, Brasil, 2019.

\begin{tabular}{lll}
\hline Variável & N & \% \\
\hline Sobrecarga dos cuidadores & & \\
Ausente (0 a 20) & 5 & 9,6 \\
Leve a moderada (21 a 40) & 9 & 17,3 \\
Moderado a severa (41 a 60) & 17 & 32,7 \\
Intensa (61 a 88) & 21 & 40,4 \\
\hline Total & $\mathbf{5 2}$ & $\mathbf{1 0 0}$ \\
\hline
\end{tabular}

Fonte: Autores (2019).

\section{Discussão}

Os resultados deste trabalho evidenciaram que, na amostra estudada, a função de cuidador é exercida majoritariamente pelas mulheres. A prevalência do sexo feminino no papel de cuidador principal é amplamente relatada na literatura nacional e internacional (Alves et al., 2019; Ferreira, Isaac, \& Ximenes, 2018; Nunes, Brito, Duarte, \& Lebrao, 2018; Pavarini et al., 2017; Six, Musomi, \& Deschepper, 2019). Este fato reflete uma concepção histórica sobre a mulher, que ainda persiste na sociedade atual, de que o ato de cuidar dos membros da família é uma atribuição naturalmente feminina (Ferreira, Isaac, \& Ximenes, 2018).

Em relação a faixa etária, constatou-se que a maioria dos cuidadores estava entre 41 a 50 anos, o que coincide com os achados de outras pesquisas (Anjos et al., 2015; Loureiro \& Fernandes, 2015). Contudo, ressalta-se que nesse estudo houve a participação de cuidadores com mais de 60 anos de idade, em uma proporção de 5,8\% (n=3). Atualmente, com o aumento da expectativa de vida, é crescente o número de cuidadores idosos prestando assistência a um outro idoso (Pereira \& Soares, 2015). A idade avançada dos cuidadores de idosos pode interferir na qualidade do cuidado e na saúde dos cuidadores, tendo em vista que esses indivíduos são menos aptos aos esforços físicos exigidos para a função, estando, desse modo, mais vulneráveis ao desenvolvimento de problemas de saúde (Nunes et al., 2018; Silva, Machado, Ferreira, \& Rodrigues, 2015).

No que se refere ao estado civil, 61,3\% dos cuidadores declararam ser casados. Esse resultado é similar ao encontrado no estudo transversal de Alves et al (2019), que ao analisar o perfil de 42 cuidadores informais de idosos dependentes no município de Vitória da Conquista, Bahia, evidenciaram que a maioria dos participantes eram casados. A presença de um companheiro pode oferecer suporte emocional aos cuidadores, contribuindo positivamente no desenvolvimento das atividades de cuidado ao idoso (Loureiro \& Fernandes, 2015).

Com relação a escolaridade, foi identificado uma predominância de cuidadores com ensino fundamental incompleto. O nível de instrução é um aspecto relevante para a função de cuidador, uma vez que a falta deste pode dificultar a administração da dieta, a compreensão das prescrições medicamentosa, bem como o entendimento das orientações e 
informações repassadas pelos profissionais de saúde, comprometendo, desta maneira, a assistência ao idoso (Santos \& Cunha, 2013; Santos-Orlandi et al., 2017).

Quanto ao grau de parentesco, percebeu-se que grande parte dos cuidadores possuíam algum vínculo familiar com o idoso, havendo uma prevalência de filhos no exercício da função de cuidador. Esse achado corrobora com dados encontrados na literatura (Lopes, Oliveira, Stigger, \& Lemos, 2020; Nardi, Sawada, \& Santos, 2013). Pode-se atribuir esta predominância ao fato do cuidado com os pais, devido a aspectos culturais e sociais, ser considerado uma responsabilidade moral dos filhos, sendo uma forma de retribuir os cuidados e carinhos recebidos dos seus progenitores (Felipe et al., 2020; Fuhrmann, Bierhals, Santos, \& Paskulin, 2015).

No que se refere ao tempo exercendo a ocupação de cuidador, o estudo mostrou que a maioria dos participantes, com $36,5 \%$, exerciam essa função há mais de quatro anos. Esse resultado é maior que o encontrado no estudo do Lopes et al. (2020) e menor que os seis anos observados na pesquisa de Felipe et al. (2020). Constatou-se ainda que 42,3\% dos cuidadores dedicam mais de 8 horas diárias de assistência ao idoso. A carga horária de trabalho elevada é, frequentemente, autorrelatada por cuidadores de idosos (Diniz et al., 2018; Gratao et al., 2012), constituindo um dos fatores de risco para a sobrecarga nesses indivíduos (Gratao et al., 2012).

Em relação ao apoio de outras pessoas na execução da tarefa de cuidador, 94,2\% dos participantes relataram não receber ajuda. De encontro a esse achado, um estudo descritivo e transversal, realizado com uma amostra de 86 cuidadores de idosos no município de São Carlos, São Paulo, evidenciou que 55,8\% dos cuidadores recebiam o auxílio de outras pessoas durante o cuidado com o idoso (Jesus, Orlandi, \& Zazzetta, 2018). O apoio de terceiros na execução das tarefas de cuidado pode possibilitar que os cuidadores disponham de tempo para realizarem outras atividades, como as de lazer, melhorando, desse modo, a qualidade de vida desses indivíduos (Alves et al., 2019; Fuhrmann et al., 2015).

Quanto ao nível de sobrecarga, observou-se que $90,4 \%$ dos cuidadores estavam sobrecarregados, sendo que 40,4\% apresentavam uma sobrecarga intensa e 32,7\% sobrecarga moderada a severa. Esse dado é superior ao encontrado em um estudo observacional realizado com 35 cuidadores informais de idosos, onde, ao aplicar a escala de sobrecarga ZBI, apenas $14,3 \%$ dos participantes estavam intensamente sobrecarregados e 25,7\% apresentavam sobrecarga moderada a severa (Diniz et al., 2018).

O nível de incapacidade funcional do idoso, as alterações na vida pessoal dos cuidadores e a quantidade de tempo destinado as funções de cuidador são fatores que contribuem para a sobrecarga dos cuidadores (Lopes et al., 2020). No presente estudo, notou-se que grande parte dos cuidadores dedicam muitas horas diárias na função de cuidador e não recebem auxílio de outras pessoas para desempenhar essa tarefa, o que pode ter contribuído para os níveis elevados de sobrecarga autorrelatados.

\section{Conclusão}

Os resultados obtidos neste estudo revelaram uma prevalência de cuidadores do sexo feminino, entre 41 a 50 anos de idade, casados, com ensino fundamental incompleto, sendo os filhos os principais responsáveis pelo cuidado com o idoso. A maioria dos cuidadores exerciam a função por mais de quatro anos, dedicando mais de oito horas diárias de assistência ao idoso e não recebiam apoio de outras pessoas no cuidado. Constatou-se ainda que grande parte dos cuidadores apresentavam algum nível de sobrecarga.

Esses achados, fornecerão informações sobre as particularidades e a realidade dos cuidadores informais de idosos dependentes do município. Além disso, poderão sensibilizar os profissionais da Atenção Básica sobre os impactos do cuidado ao idoso dependente na saúde de seus cuidadores, contribuindo para o planejamento de estratégias que capacitem esses indivíduos na assistência ao idoso e previnam o aparecimento de problemas de saúde relacionados ao trabalho, assegurando, 
desse modo, a qualidade de vida e a saúde do cuidador de idosos, que, frequentemente, é negligenciado pelos serviços de saúde.

Contudo, ressalta-se que o presente estudo apresenta limitações que devem ser consideradas. Primeiro, a escolha das UBS foi determinada por conveniência, não havendo uma investigação em in loco para verificar as áreas com maior concentração de idosos dependentes e de seus cuidadores. Segundo, o fato de o estudo ter sido realizado em apenas quatro UBS impede a generalização dos resultados para outras áreas do município, dificultando o desenvolvimento de ações educativas, por meio de palestras e cursos de capacitação, que minimizem os impactos ocupacionais na saúde dos cuidadores de idosos dessa região.

\section{Referências}

Alves, B. S., Oliveira, A. S., Santana, E. S., Chaves, R. N., Marinho, M. S., \& Reis, L. A. (2019). Caracterização dos cuidadores informais de idosos dependentes quanto aos aspectos demográficos e de saúde. Revista de Saúde Coletiva da UEFS, 9, 113-118. http://dx.doi.org/10.13102/rscdauefs.v9i0.3684.

Anjos, K. F., Boery, R. N. S. O., Pereira, R., Pedreira, L. C., Vilela, A. B. A., Santos, V. C., \& Rosa, D. O. S. (2015). Associação entre apoio social e qualidade de vida de cuidadores familiares de idosos dependentes. Ciências \& Saúde Coletiva, 20(5), 1321-1330. https://doi.org/10.1590/141381232015205.14192014 .

Batista, M. P. P., Almeida, M. H. M., \& Lancman, S. (2014). Cuidadores formais de idosos: contextualização histórica no cenário brasileiro. Revista Brasileira de Geriatria e Gerontologia, 17(4), 879-885. https://doi.org/10.1590/1809-9823.2014.13148.

Boaventura, L. C., Borges, H. C., \& Ozaki, A. H. (2016). Avaliação da sobrecarga do cuidador de pacientes neurológicos cadeirantes adultos. Ciências \& Saúde Coletiva, 21(10), 3193-3202. https://doi.org/10.1590/1413-812320152110.15202016.

Costa, T. F., Martins, K. P., Bezerra, T. A., Valdevino, S. C., Pimenta, J. P., \& Costa, K. N. F. M. (2019). Estrutura fatorial da Escala Zarit Burden Interview em cuidadores de pacientes com acidente vascular encefálico. Revista Enfermagem Atual In Derme, 87(25), 1-10. https://revistaenfermagematual.com.br/index.php/revista/article/view/206/107.

Couto, A. M., Castro, E. A. B., \& Caldas, C. P. (2016). Vivências de ser cuidador familiar de idosos dependentes no ambiente domiciliar. Revista Rene, 7(1), 76-85. 10.15253/2175-6783.2016000100011.

Diniz, M. A. A., Melo, B. R. S., Neri, K. H., Casemiro, F. G., Figueiredo, L. C., \& Gaioli, C. C. L. O. et al. (2018). Estudo comparativo entre cuidadores formais e informais de idosos. Ciências \& Saúde Coletiva, 23(11), 3789-3798. http://dx.doi.org/10.1590/1413-812320182311.16932016.

Felipe, S. G. B., Oliveira, C. E. S., Silva, C. R. D. T., Mendes, P. N., Carvalho, K. M., \& Silva-Junior, F. L. et al.(2020). Anxiety and depression in informal caregivers of dependent elderly people: an analytical study. Rev. Brasileira de Enfermagem, 73(1), 1-8. http://dx.doi.org/10.1590/0034-7167-2019-0851.

Fernandes, B. C. W., Ferreira, K. C. P., Marodin, M. F., Val, M. O. N., \& Fréz, A. R. (2013). Influência das orientações fisioterapêuticas na qualidade de vida e na sobrecarga de cuidadores. Fisioterapia em Movimento, 26(1), 151-158. https://doi.org/10.1590/S0103-51502013000100017.

Ferreira, C. R., Isaac, L., \& Ximenes, V. S. (2018). Cuidar de idosos: um assunto de mulher?. Estudos Interdisciplinares em Psicologia, 9(1), 108-125. 10.5433/2236-6407.2016v9n1p108.

Fuhrmann, A. C., Bierhals, C. C. B. K., Santos, N. O., \& Paskulin, L. M. G. (2015)Associação entre a capacidade funcional de idosos dependentes e a sobrecarga do cuidador familiar. Revista Gaúcha de Enfermagem, 36(1), 14-20. https://doi.org/10.1590/1983-1447.2015.01.49163.

Gratao, A. C. M., Vendrúscolo, T. R. P., Talmelli, L. F. S., Figueiredo, L. C., Santos, J. L. F., \& Rodrigues, R. A. P. (2012) Sobrecarga e desconforto emocional em cuidadores de idosos. Texto \& Contexto Enfermagem, 21(2), 304-312. https://www.scielo.br/pdf/tce/v21n2/a07v21n2.

Instituto Brasileiro de Geografia e Estatística. (2016). Síntese de indicadores sociais: uma análise das condições de vida da população brasileira 2016. Rio de Janeiro: IBGE. https://biblioteca.ibge.gov.br/visualizacao/livros/liv98965.pdf.

Instituto Brasileiro de Geografia e Estatística. (2010). Projeção da população do Brasil e das Unidades da Federação. https://www.ibge.gov.br/apps/populacao/projecao/index.html.

Jesus, I. T. M., Orlandi, A. A. S., \& Zazzetta, M. S. (2018). Sobrecarga, perfil e cuidado: cuidadores de idosos em vulnerabilidade social. Revista Brasileira de Geriatria e Gerontologia, 21, 119-209. https://doi.org/10.1590/1981-22562018021.170155.

Lopes, C. C., Oliveira, G. A., Stigger, F. S., \& Lemos, A. T. (2020). Associação entre a ocorrência de dor e sobrecarga em cuidadores principais e o nível de independência de idosos nas atividades de vida diária: estudo transversal. Caderno Saúde Coletiva, 28(1), 98-106. http://dx.doi.org/10.1590/1414462x202028010184.

Loureiro, L. S. N., \& Fernandes, M. G. M. (2015). Perfil do cuidador familiar de idosos dependentes em convívio domiciliar. Revista de Pesquisa Cuidado é Fundamental Online, 7,145-154. https://www.redalyc.org/pdf/5057/505750949011.pdf.

Maronesi, L. C., Silva, N. R., Cantu, S. O., \& Santos, A. R. (2014). Indicadores de estresse e sobrecarga em cuidadores formais e informais de pacientes oncológicos. Estudos \& Pesquisas em Psicologia, 14(3), 877-892. https://doi.org/10.12957/epp.2014.13889. 
Research, Society and Development, v. 10, n. 6, e47210616061, 2021

(CC BY 4.0) | ISSN 2525-3409 | DOI: http://dx.doi.org/10.33448/rsd-v10i6.16061

Medeiros, K. K, Pinto Junior, E. P, Bousquat, A., \& Medina, M. G. (2017). O desafio da integralidade no cuidado ao idoso, no âmbito da Atenção Primária à Saúde. Saúde Debate, 41(3), 288-295. https://doi.org/10.1590/0103-11042017s322.

Mendes, P. N., Figueiredo, M. L. F., Santos, A. M. R., Fernandes, M. A, \& Fonseca, R. S. B. (2019). Sobrecargas física, emocional e social dos cuidadores informais de idosos. Acta Paulista de Enfermagem, 32(1), 87-94. https://doi.org/10.1590/1982-0194201900012.

Nardi, E. F. R., Sawada, N. O., \& Santos, J. L. F. (2013). Associação entre a incapacidade funcional do idoso e a sobrecarga do cuidador familiar. Rev. Latino-americana de Enfermagem, 21(5), 1-8. http://dx.doi.org/10.1590/S0104-11692013000500012.

Nunes, D. P., Brito, T. R. P., Duarte, Y. A. O., \& Lebrao, M. L. (2018). Cuidadores de idosos e a tensão excessiva associada ao cuidado: evidências do Estudo Sabe. Revista Brasileira de Epidemiologia, 21(2), 1-14. 10.1590/1980-549720180020.supl.2.

Pavarini, S. C. I., Neri, A. L., Brigola, A. G., Ottaviani, A. C., Souza, E. N., Rossetti, E. S., \& Terassi, M. et al. (2017). Idosos cuidadores que moram em contextos urbanos, rurais e de alta vulnerabilidade social. Revista da Escola de Enfermagem da USP, 51, 1-7. http://dx.doi.org/10.1590/S1980220X2016040103254.

Pedreira, L. C., \& Oliveira, M. A. S. (2012). Cuidadores de idosos dependentes no domicílio: mudanças nas relações familiares. Revista Brasileira de Enfermagem, 65(5), 730-736. https://www.scielo.br/pdf/reben/v65n5/03.pdf.

Pereira, L. S. M., \& Soares, S. M. (2015). Fatores que influenciam a qualidade de vida do cuidador familiar do idoso com demência. Ciências \& Saúde Coletiva, 20(12), 3839- 3851. https://doi.org/10.1590/1413-812320152012.15632014.

Santos, G. S., \& Cunha, I. C. K. O. (2013). Perfil sociodemográfico de cuidadores familiares de idosos residentes em uma área de abrangência da Estratégia Saúde da Família no município de São Paulo. Saúde Coletiva, 10(60), 47-53. https://www.redalyc.org/pdf/842/84228212008.pdf.

Santos-Orlandi, A. A., Brito, T. R. P., Ottaviani, A. C., Rossetti, E. S., Zazzetta, M. S., \& Gratão, A. C. M. (2017). Perfil de idosos que cuidam de outros idosos em contexto de alta vulnerabilidade social. Escola Anna Nery, 21(1), 1-8: https://doi.org/10.5935/1414-8145.20170013.

Silva, I. L. S., Machado, F. C. A., Ferreira, M. A. F., \& Rodrigues, M. P. (2015). Formação profissional de cuidadores de idosos atuantes em instituições de longa permanência. Holos, 8, 342-356. https://doi.org/10.15628/holos.2015.3215.

Six, S., Musomi, S., \& Deschepper, R. (2019). Are the Elderly Perceived as a Burden to Society? The Perspective of Family Caregivers in Belgium and Kenya: A Comparative Study. Journal of Transcultural Nursing, 30(2), 124-131. 10.1177 / 1043659618784358. 